\title{
Meth-DOP-PCR: an assay for the methylation profiling of trace amounts of DNA extracted from bodily fluids
}

\author{
Angela Di Vinci, Ilaria Gelvi, Barbara Banelli, Ida Casciano, Giorgio Allemanni \\ and Massimo Romani \\ Experimental Oncology 'C', Istituto Nazionale per la Ricerca sul Cancro (IST-GE), Genova, Italy
}

\begin{abstract}
Cancer cells release their DNA into the patient's bodily fluids and cancer-specific signatures can be recognized in the circulating DNA. The aberrant methylation of CpG-rich regions in gene promoter sequences is an early marker of cell transformation whose specificity and optimal sensitivity can be achieved by assessing the methylation status of multiple genes ('methylation profiling'). Most of the current technologies for methylation analysis rely upon the combination of chemical conversion of the DNA and PCR analysis for the detection of methylated and unmethylated alleles. However, the small amount of circulating DNA, and its fragmentation, dramatically reduces the template DNA molecules making difficult the methylation profiling. To overcome this limitation, we have developed the Meth-DOP-PCR assay, a combination between a modified degenerate oligonucleotide primed PCR (DOP-PCR) and methylation-specific PCR (MSP), for the high-throughput methylation analysis of trace-amount of circulating DNA. We have demonstrated the concordance between Meth-DOP-PCR and MSP and shown the application of this technique for the methylation analysis of DNA extracted from the serum of lung cancer patients. We have estimated that through this procedure it is possible to obtain at least a 25-fold increase of the number of determinations allowing the methylation profiling from less than $1 \mathrm{ml}$ of serum. Thus, Meth-DOP-PCR appears as a simple, cost-effective and efficient technique, for the development of novel methylation-based diagnostic assays. Laboratory Investigation (2006) 86, 297-303. doi:10.1038/labinvest.3700384; published online 30 January 2006
\end{abstract}

Keywords: methylation; circulating DNA; early diagnosis; lung cancer

Early diagnosis, along with prevention and optimal treatment, is the most critical challenge for improving the overall cancer burden. Indeed disease extension at the time of diagnosis is one of the most important prognosis factors in solid tumors and monitoring the efficacy of the therapy could help the physicians in defining better strategies for the management of the patient.

The early diagnosis by sophisticated imaging techniques and histopathological examination of deep, small lesions that can be reached with difficulty, such as those of lung cancer, is impaired by the iatrogenic risk of accessing the affected organ and by the cost of these procedures that reduces the opportunity of screening in high-risk populations. Therefore, for most types of cancer, the development of specific biomarkers and sensitive assays is the best

Correspondence: Dr M Romani, MD, Experimental Oncology 'C', Istituto Nazionale per la Ricerca sul Cancro (IST-GE), Largo Rosanna Benzi 10, Genova 16132, Italy.

E-mail: massimo.romani@istge.it

Received 25 September 2005; revised 24 November 2005; accepted 27 November 2005; published online 30 January 2006 hope for early detection. In this respect, an enormous interest has been recently raised by the discovery that cancer cells release their DNA into the patient's bodily fluids and that cancer-specific signatures, like point mutations and DNA methylation errors, can be recognized in the circulating DNA by PCR-based technologies that are the core for the development of fast and accurate assay for the early detection of primary cancer or of their recurrence. ${ }^{1-5}$ Furthermore, although the identification of DNA methylation markers predictive of the response to chemotherapy is just beginning, several studies have shown the association between specific methylation aberrations and the efficacy of chemotherapy in cancer patients. ${ }^{6-8}$

Alteration of the methylation status of selected CpG-rich promoter sequences is an early event of cell transformation that influences gene expression and thus is a particularly promising cancer biomarker for early diagnosis (reviewed in Baylin and Herman, ${ }^{9}$ Verma and Srivatsava, ${ }^{10}$ and Esteller ${ }^{11}$ ). In this respect, it must be considered that methylation may act as a relatively simple 'yes or no' signal for the presence of tumor DNA or for tumor recurrence. Therefore, the optimization of the techniques for its 
detection as well as the availability of a panel of relevant target sequences is a problem of tremendous biomedical importance. Moreover, an important advantage of DNA methylation analysis is that, in contrast to point mutations that can occur at variable locations within a gene promoter hypermethylation involves the same sequence in all patients and all tumors, thereby simplifying the assay methods enormously.

The analytical sensitivity of methylation assays (ie the ability of detecting minimal amounts of methylated target in a biological sample) is quite high; however, it must be considered that the tumor prevalence of the methylation markers identified so far is less than $100 \%$, thus the utilization of this strategy in a clinical environment requires that multiple markers are utilized in order to achieve optimal clinical sensitivity and specificity.

The current technologies for the methylation analysis rely upon the sodium bisulfite modification of DNA that results in the conversion of all Cytosine, not followed by a Guanine, into Thymine. Cytosine preceding Guanine (CpG) are converted into T only if they are unmethylated, and remain as $\mathrm{C}$ when methylated. ${ }^{12}$

The methylation status of a given sequence is then determined by methylation-specific PCR (MSP) or by sequencing or by restriction enzyme analysis after PCR amplification of the bisulfite-modified DNA (BRE). ${ }^{12-14}$

The most serious limiting factor of the methylation analysis on the DNA extracted from bodily fluids is the small amount of starting material that generally is in the range of $50 \mathrm{ng} / \mathrm{ml}$ but that can also be much lower. ${ }^{14}$ Moreover, the inevitable fragmentation and loss of material during the modification and post-treatment steps dramatically reduces the number of DNA molecules that can act as an efficient template for PCR amplification further reducing the possibility of constructing a representative profile of methylation from circulating DNA.

We report the development and validation on clinical samples of the Meth-DOP-PCR, a modified degenerate oligonucleotide-primed PCR amplification (DOP-PCR) that is combined with MSP, for the sensitive and specific detection of methylation aberrations in circulating DNA.

Although Meth-DOP-PCR can be applied to any type of cancer, we have focused our analysis on lung cancer, a leading cause of cancer mortality throughout the world, whose impact could be effectively reduced by early diagnosis and screening programs carried out by simple non-invasive assays.

\section{Materials and methods}

\section{Sample Collection}

Blood samples from non small-cell lung cancer patients were drawn before surgery. The blood was centrifuged at 2000 r.p.m. for $10 \mathrm{~min}$ at room temperature and serum aliquots were stored at $-80^{\circ} \mathrm{C}$ until use. Matching tumor samples were snap frozen in liquid nitrogen and stored at $-80^{\circ} \mathrm{C}$ immediately after surgery.

Cell lines U937, HL60, ACN and HTLA were utilized as reference standard for methylation or unmethylation. The methylation status of the genes considered in this study in the reference set of cell lines was previously determined at least by two methods.

\section{DNA Extraction and Modification}

DNA was extracted from $0.4-2 \mathrm{ml}$ of serum using QIAmp DNA blood Midi kit (Qiagen, Hilden, Germany) according to the manufacturer's instructions.

Tumor and cell lines DNA was obtained using by standard Proteinase K/SDS digestion overnight at $55^{\circ} \mathrm{C}$ followed by phenol/chloroform extraction and ethanol precipitation. The DNA was modified by sodium bisulfite treatment as described by Frommer et $a l^{12}$ and Herman et $a .^{13}$

\section{DOP-PCR Amplification}

DOP-PCR amplification was performed utilizing a two-step PCR at different stringency conditions.

The first step (low-stringency amplification) was carried out in a volume of $10 \mu \mathrm{l}$ in a reaction mixture containing $4 \mathrm{U}$ of Taq polymerase (ThermoSequenase, Amersham, Cleveland, OH, USA), reaction buffer, $0.2 \mathrm{mM}$ dNTPs and $0.2 \mu \mathrm{M}$ of each of the following DOP primers: 5'-CCGACTCGAAHHHHH HATATAA-3' (methylated primer) and 5'-CCAACT CAAAHHHHHHATATAA-3' (unmethylated primer) (where H: A, C or T). After an initial denaturation step of $3 \mathrm{~min}$ at $95^{\circ} \mathrm{C}$, the DNA was amplified for four cycles followed by a final extension step of $10 \mathrm{~min}$ at $74^{\circ} \mathrm{C}$ and $5 \mathrm{~min}$ at $25^{\circ} \mathrm{C}$. Each cycle consisted of $1 \mathrm{~min}$ at $94^{\circ}, 1 \mathrm{~min}$ at $25^{\circ}, 3 \mathrm{~min}$ transition from $25^{\circ}$ to $74^{\circ} \mathrm{C}$ and $2 \mathrm{~min}$ at $74^{\circ} \mathrm{C}$. The second PCR step (stringent amplification) was conducted in a reaction volume of $50 \mu \mathrm{l}$ containing $2.5 \mathrm{U}$ of Platinum Taq polimerase (Invitrogen), reaction buffer, $0.2 \mathrm{mM}$ dNTPs, $1.5 \mathrm{mM} \mathrm{MgCl}_{2}$ and $0.2 \mu \mathrm{M}$ of each of DOP primers used in the first step.

After an initial denaturation step $\left(3 \mathrm{~min}\right.$ at $\left.95^{\circ} \mathrm{C}\right)$, the DNA was amplified for 35 cycles $\left(1 \mathrm{~min}\right.$ at $94^{\circ} \mathrm{C}$, $1 \mathrm{~min}$ at $56^{\circ} \mathrm{C}, 2 \mathrm{~min}$ at $72^{\circ} \mathrm{C}$ ) followed by a final extension at $72^{\circ} \mathrm{C}$.

\section{Methylation Analysis}

The methylation status of target genes was determined by bisulfite restriction enzyme (BRE) analysis and by standard MSP. ${ }^{13,15}$

The sequence of the primers utilized for BRE and MSP and their annealing temperatures is reported in 
Table 1. For MSP, 1-5 $\mu$ l of DOP product was amplified in $50 \mu \mathrm{l}$ reaction volume containing $0.6 \mu \mathrm{M}$ of primers specific for the methylated or the unmethylated DNA, 2.5 U of Platinum Taq polymerase (Invitrogen), reaction buffer, $0.2 \mathrm{mM}$ dNTPs and $1.5 \mathrm{mM} \mathrm{MgCl}_{2}$.

After an initial denaturation step of $2 \mathrm{~min}$ at $94^{\circ} \mathrm{C}$, the DNA was amplified for 35-40 cycles each consisting of $30 \mathrm{~s}$ at $94^{\circ} \mathrm{C}, 30 \mathrm{~s}$ at the annealing temperature (Table 1) and $1 \mathrm{~s}$ at $72^{\circ} \mathrm{C}$ followed by a final extension step of $10 \mathrm{~s}$ at $72^{\circ} \mathrm{C}$.

The PCR products were resolved on a $3 \%$ Agarose-1000 Gel (Invitrogen, Milano, Italy). For $\mathrm{BRE}$, the purified products were digested with Rsa I before gel analysis.

\section{Results}

\section{Overview and Design of the Technique}

Genomic DNA, after bisulfite modification was either utilized directly for MSP assays or processed for Meth-DOP-PCR (Figure 1a).

To develop Meth-DOP-PCR, we amplified the sodium bisulfite-modified DNA with two degenerate oligonucleotide primers designed to amplify the methylated and the unmethylated DNA. The original DOP-PCR ${ }^{16}$ relies upon the use of a single primer consisting in a $3^{\prime}$ region corresponding to an examer repeated approximately $10^{6}$-fold in the human genome followed by a degenerate sequence that stabilizes the annealing. This strategy was successfully utilized for the whole-genome amplification for microsatellite genotyping ${ }^{17}$ and SNP analysis. ${ }^{18}$

Starting from the standard DOP-PCR primer we have designed the two Meth-DOP-PCR-modified oligonucleotides by substituting all $G$ with $A$ for the amplification of the unmethylated DNA, while in the primer for the methylated target only the $\mathrm{G}$ not following $\mathrm{C}$ nucleotides were substituted with $\mathrm{A}$.
The amplification of bisulfite-modified DNA with the DOP primers consists in a two-step reaction at different annealing temperatures. In the first

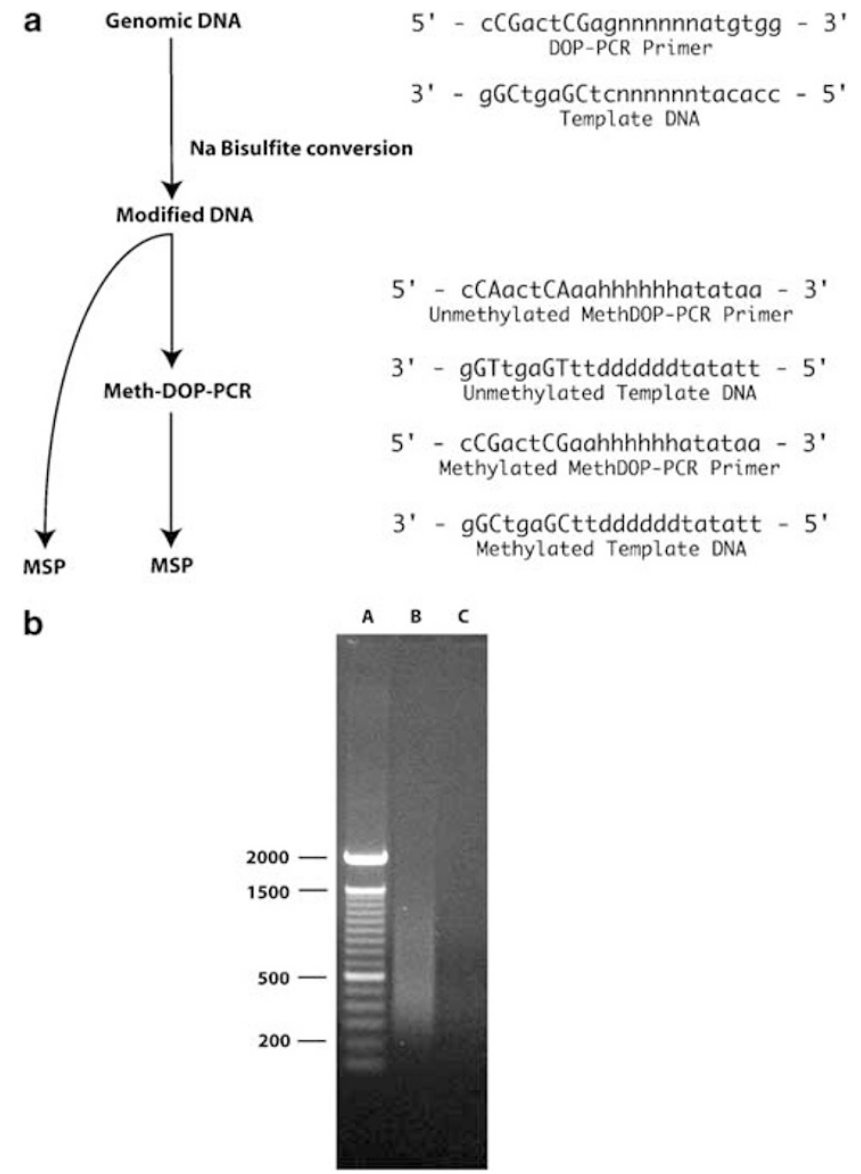

Figure 1 The Meth-DOP-PCR assay. (a) Outline of a typical MethDOP-PCR experiment compared to direct MSP. The sequence of the DOP primer for native DNA and those for the modified DNA are indicated. $\mathrm{N}=\mathrm{A}, \mathrm{C}, \mathrm{G}, \mathrm{T} ; \mathrm{H}=\mathrm{A}, \mathrm{C}, \mathrm{T} ; \mathrm{D}=\mathrm{A}, \mathrm{G}, \mathrm{T}$. (b) Requirement for the low-stringency amplification step. Lane A: $100 \mathrm{bp}$ ladder. The modified DNA was subjected to Meth-DOPPCR as described in Materials and methods (lane B) or omitting the enzyme only in the low stringency step (lane C).

Table 1 Primers and PCR conditions for MSP analysis

\begin{tabular}{|c|c|c|c|c|}
\hline Gene & Set & $S N$ & $A S N$ & $\mathrm{Ta}$ \\
\hline \multirow[t]{2}{*}{$R A S S F 1 A$} & $\mathrm{M}$ & gggaggcgttgaagtcggg & caacgcgcccaacgaatac & 62 \\
\hline & UM & gaggtgttgaagttggggtttg & cсcаacacacccaacaaat & 68 \\
\hline \multirow[t]{2}{*}{$C D K N 2 A$} & $\mathrm{M}$ & ttattagagggtggggcggatcgc & gaccccgaaccgcgaccgtaa & 70 \\
\hline & UM & ttattagagggtggggtggattgt & caaccсcаaасcасаaсcataa & 62 \\
\hline \multirow[t]{2}{*}{ DCR2 } & M & gggataaagcgtttcgatc & cgacaacaaaaccgcg & 60 \\
\hline & UM & ttggggataaagtgttttgatt & аaассаасаасааaассаса & 60 \\
\hline \multirow[t]{2}{*}{ TP73 (TA) } & M & cgggttttgtaggagcgac & acgcctttttaacccgacg & 68 \\
\hline & UM & ttgttgggttttgtaggagtgatgt & acaаacattaacacctttttaacccaаca & 68 \\
\hline \multirow[t]{3}{*}{$\operatorname{TP73}(\Delta N)$} & $\mathrm{M}$ & gttgtcgggcggttacgatc & tcacacctaccgtaacgaaataccg & 63 \\
\hline & UM & ggtttatgttgttgggtggttatgattg & cacatcacacctaccataacaaaataccatac & 63 \\
\hline & BRE & ttagttgatagaattaagggagatgg & aaaaaatacccctctaaaccctaca & 59 \\
\hline \multirow[t]{2}{*}{ MGMT } & $\mathrm{M}$ & tttcgacgttcgtaggttttcgc & gcactcttccgaaaacgaaacg & 59 \\
\hline & UM & tttgtgttttgatgtttgtaggtttttgt & actccacactcttccaaaaасаaаaca & 59 \\
\hline
\end{tabular}

SN: sense primer; ASN: anti sense primer; Ta: annealing temperature; BRE: bisulfite restriction enzyme analysis; M: methylated; UM: unmethylated. 
low-stringency step, conducted at $25^{\circ} \mathrm{C}$, the target DNA is non-specifically and uniformly amplified to increase the yield of template DNA and to add tags at the fragment ends. In the second, high-stringency step, the tagged DNA is exponentially amplified.

The initial low-stringency amplification, where a partial hybridization between primers and target DNA occurs, is essential for the efficient generation of template necessary for the subsequent highstringency amplification. Indeed, as shown in Figure 1b, a DNA smear ranging from 200 to $1500 \mathrm{bp}$ is detectable after the two-step PCR but not if the low-stringency step is omitted.

Although we cannot formally demonstrate that all CpG-containing genes are represented in the MethDOP-amplified DNA, all the randomly selected genes analyzed in the present study could be detected in the methylation-specific assay.

To determine the methylation status of a specific target sequence, aliquots of the DOP-amplified DNA were subjected to PCR utilizing primers and reaction conditions identical to those employed for the direct MSP assay.

The variable recovery of the DNA after the modification step did not allow the precise calculation of the absolute yield of template obtained by Meth-DOP-PCR. To roughly estimate the extent of enrichment of template DNA for MSP analysis, we performed a methylation analysis for the TA-p73 gene with serial dilutions of DNA from cell lines U937 and HL60. In these cells the TA-p73 promoter is completely methylated and unmethylated, respectively. ${ }^{19}$ Briefly, serial dilutions of DNA ranging from 500 to $7.8 \mathrm{ng}$ (Figure 2) were modified with sodium bisulfite in separate reactions and dissolved in $30 \mu \mathrm{l}$. Two microliter aliquots of the modified DNA that, assuming no loss of material, correspond to $33-0.51 \mathrm{ng}$ of modified DNA, were subjected to Meth-DOP-PCR in a final volume of $50 \mu \mathrm{l}$. Two microliter of the amplicons generated by Meth-DOP, derived from the amplification of 1.32-0.02 ng of the starting DNA, were utilized for MSP. In parallel experiments $2 \mu \mathrm{l}$ of the modified DNA were directly subjected to MSP with the same primer set. As internal control, we performed a mock Meth-DOP experiment, by omitting the Taq Sequenase in the reaction, followed by MSP.

The data reported in Figure 2, show that, under the experimental conditions utilized in this assay, the amplification products derived from Meth-DOPPCR are clearly detectable at least up to $15 \mathrm{ng}$ of DNA (lane F) and that detectable signals are still present at $7.8 \mathrm{ng}$ (lane G). Furthermore, Meth-DOPPCR requires 25-fold less template to generate results comparable to those of MSP thus us allowing to perform multiple assays and, in turn, the methylation profiling from trace amount of DNA. Moreover, within the lower range of DNA concentrations found in the serum of cancer patients (62.5$7.8 \mathrm{ng}),{ }^{14}$ Meth-DOP-PCR generates stronger signals as compared to MSP.

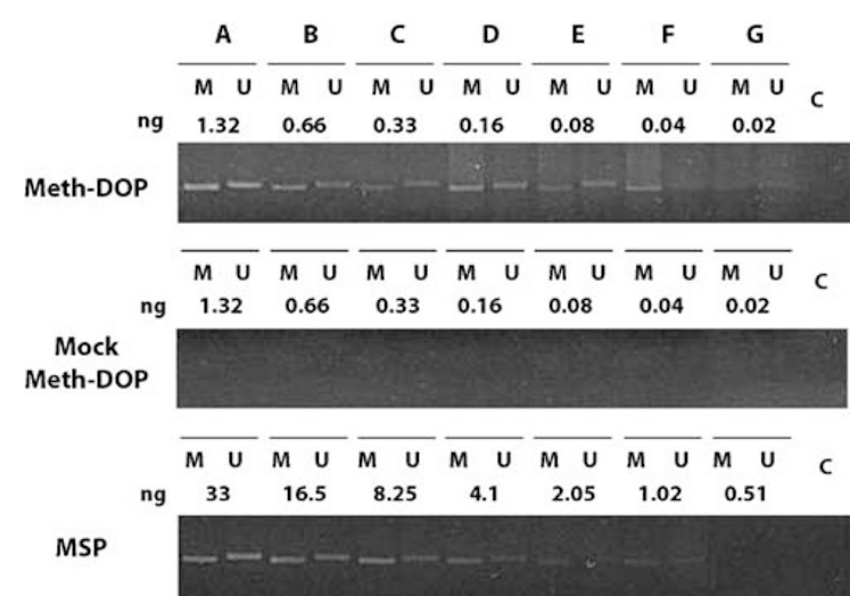

Figure 2 Methylation analysis of the TA-p73 gene in cell lines U937 (methylated, M) and HL60 (unmethylated, U) after distinct modification reactions conducted with decreasing amounts of genomic DNA. Five hundred (lane A) 250, (lane B) 125 (lane C), 62.5 (lane D), 31.2 (lane E), 15.6 (lane F) and 7.8 (lane G) ng of genomic DNA were modified with sodium bisulfite and diluted in $30 \mu \mathrm{l}$ of water. Aliquots of $2 \mu \mathrm{l}$ (theoretically corresponding to 33, $16.5,8.25,4.1,2.05,1.02$ and $0.51 \mathrm{ng}$ of the original DNA) were subjected to Meth-DOP-PCR (upper panel) or to MSP (lower panel). $1 / 25$ of the Meth-DOP-PCR product (corresponding to $1.32,0.66,0.33,0.16,0.08,0.04,0.02 \mathrm{ng}$, of the original DNA) was utilized for the subsequent MSP with the TA-p73 set of primers. In parallel experiments, $2 \mu \mathrm{l}$ of modified DNA were mock amplified by Meth-DOP-PCR in reactions not containing the enzymes (middle panel).

As expected no amplification products were detectable in the mock Meth-DOP samples indicating that the template utilized for Meth-DOP is not sufficient for the direct analysis by MSP alone.

\section{Fidelity of Meth-DOP-PCR}

One of the crucial aspects of any PCR-based protocol of DNA enrichment is the fidelity of the amplification. Although the bisulfite modification followed by Meth-DOP-PCR is a rather straightforward procedure, it might also generate artefacts because of the possible bias in the amplification with the degenerate primers. To test the fidelity of MethDOP-PCR amplification, we have determined the methylation status of the $\Delta N-p 73$ gene promoter in mixtures containing different ratios of modified DNA from cell lines U937 and ACN. In these cell lines the $\Delta N-p 73$ promoter is totally methylated and unmethylated, respectively. The comparison of the methylation status determined by MSP or MethDOP-PCR was performed by BRE to detect newly formed restriction enzyme recognition sites created by bisulfite modification in the methylated DNA.

As shown in Figure 3, although some differences in the product yield were apparent, we did not observe remarkable differences in the pattern of methylation determined by bisulfite/restriction enzyme analysis alone ${ }^{15}$ or after Meth-DOP-PCR. 


\section{Accuracy of Meth-DOP-PCR}

To determine the concordance between MSP and Meth-DOP-PCR, we first assessed the methylation status of six genes (TA-p73, $\Delta N-p 73, C D K N 2 A$, RASSF1A, MGMT and DCR2) by MSP and by Meth-DOP-PCR in cell lines where the methylation status of these target genes was previously demonstrated in independent experiments (Banelli

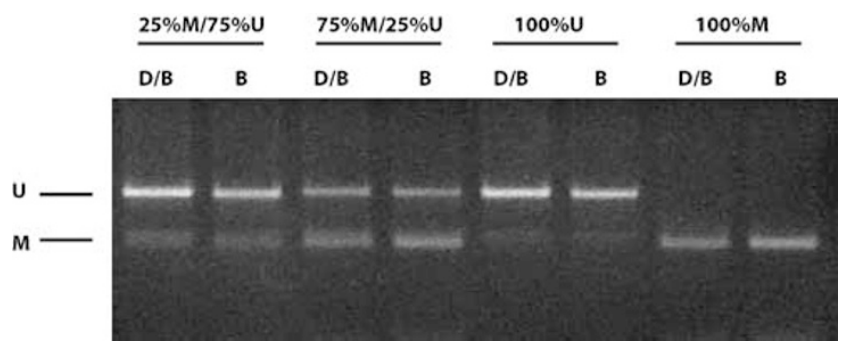

Figure 3 Fidelity of Meth-DOP-PCR. The methylation status of the $\Delta N-p 73$ promoter was determined by BRE before (B) and after (D/B) Meth-DOP-PCR. The DNA utilized in this analysis derived from U937 and ACN, two cell lines where this promoter is completely methylated and unmethylated, respectively. BRE analysis was performed with primer sets that do not discriminate between methylated and unmethylated sequence. The methylation level was determined by digestion with Rsa I, a restriction enzyme whose recognition sites is created by bisulfite modification in the methylated DNA only. U indicates the uncleaved amplification band corresponding to the non-methylated target, $\mathrm{M}$ is the band originated by restriction enzyme digestion. et $a{ }^{19,21} \mathrm{Di}$ Vinci et $a l^{20}$ and our unpublished observations). As shown in Figure 4, the six genes considered in this analysis presented an identical methylation status after MSP or Meth-DOP-PCR indicating that the results obtained by the two techniques are qualitatively concordant.

We next compared the methylation status of $M G M T$ and RASSF1A genes from frozen lung cancer fragments by Meth-DOP-PCR or by MSP alone and, also in this case, we have observed the concordance between the two techniques (Figure 5).

\section{Amplification of Circulating DNA by Meth-DOP-PCR}

The results obtained in the experiments described above, demonstrated that using Meth-DOP-PCR it is possible to obtain the representative amplification of bisulfite-modified DNA from cell lines and tissue samples for the subsequent determination of the methylation status of multiple genes. The next step for the validation of the technique was to determine if Meth-DOP-PCR could amplify trace amounts of free tumor DNA present in bodily fluids. As shown in Figure 6a, when we applied the procedure to the DNA extracted from the serum of lung cancer patients, we observed bands corresponding to the methylated or unmethylated target in the four reference gene promoter sequences considered (MGMT, RASSF1A, DCR2 and CDKN2A) demon-

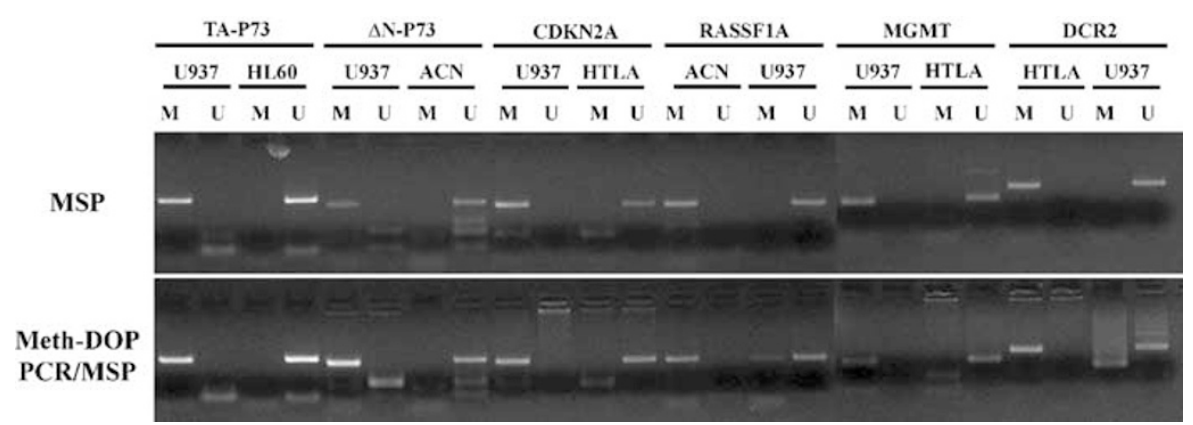

Figure 4 Comparison between Meth-DOP-PCR and MSP. Methylation analysis of six gene promoter sequences determined by MSP and Meth-DOP-PCR in cell lines of different origins. The methylation status of these reference promoters and cell lines was previously determined by sequencing.
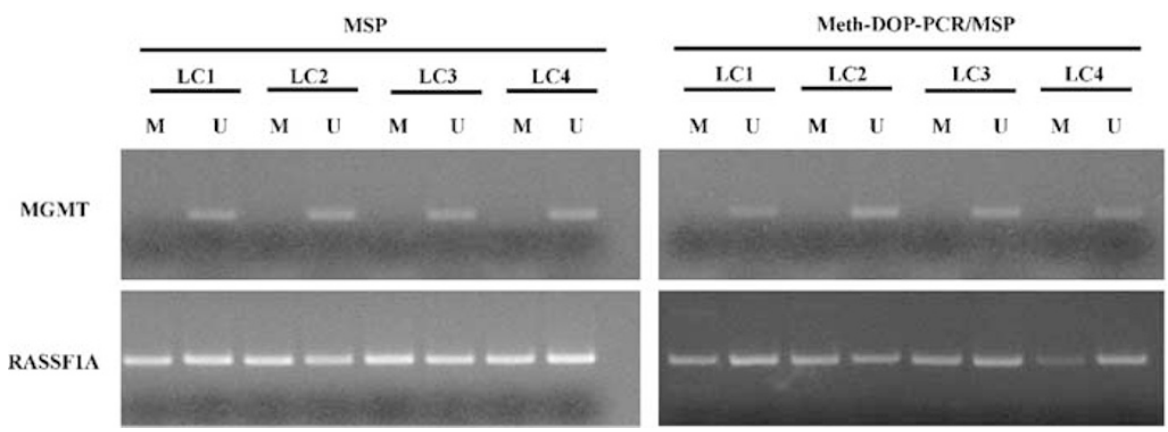

Figure 5 Comparison between Meth-DOP-PCR and MSP in tumor samples. Methylation analysis of MGMT and RASSF1A gene promoter sequences determined by MSP and Meth-DOP-PCR in four lung cancer samples. 
strating the feasibility of amplifying circulating DNA by Meth-DOP-PCR.

The variable concentration of circulating DNA makes it difficult to predict the minimal amount of serum required for Meth-DOP-PCR. However, we have observed that the lower practical volume of serum from which DNA can be successfully extracted and utilized for the construction of the methylation profile is approximately $0.4 \mathrm{ml}$.

a

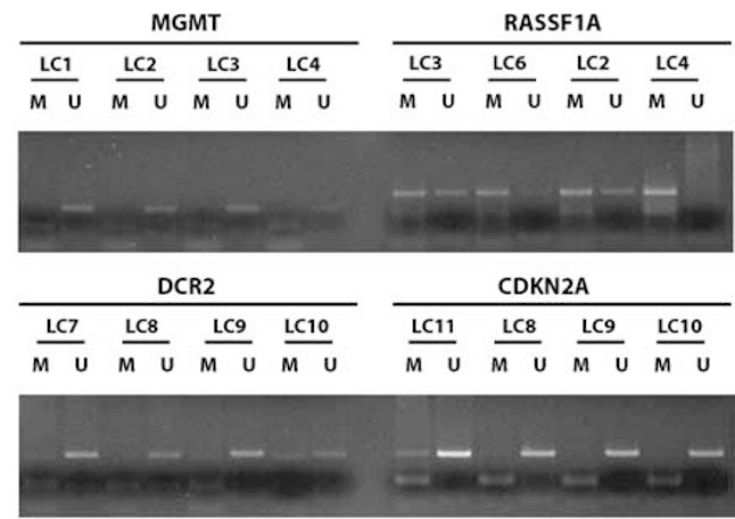

b

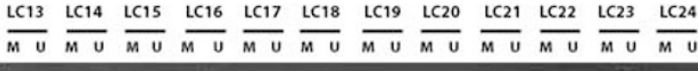

Meth-DOP

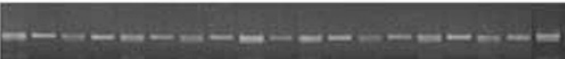

MSP

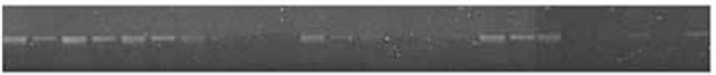

Figure 6 Meth-DOP-PCR in serum DNA. (a) Methylation analysis of MGMT, RASSF1A, DCR2 and CDKN2A gene promoter sequences determined by Meth-DOP-PCR in the circulating DNA from four lung cancers patients. (b) Comparison between Meth-DOP-PCR and MSP for the analysis of the methylation status of the RASSF1A gene in 12 serum samples from lung cancer patients.
To evaluate if Meth-DOP-PCR had an advantage over MSP alone we determined the methylation status of the RASSF1A gene in circulating DNA in 12 lung cancer patients. For each serum, equal amount of DNA were amplified by Meth-DOP-PCR or by MSP. As shown in Figure 6b, the efficiency of amplification appears considerably higher in the samples subjected to Meth-DOP-PCR. Indeed, the methylation pattern in the DNA samples amplified by MSP, the methylation status was not or only partially detectable in six out of 12 cases. Conversely, bands corresponding to the methylated and to the unmethylated alleles were readily detectable after Meth-DOP-PCR in all samples.

Finally, having established that Meth-DOP-PCR can efficiently enrich for target sequences the circulating DNA, we utilized this technique to determine the methylation status of MGMT and RASSF1A genes in a group of paired samples of serum and tumor DNA from lung cancer patients (Figure 7). We have observed that the MGMT promoter was unmethylated in each of these tissue and serum DNA samples and that the RASSF1A gene promoter was methylated both in primary cancers and in the corresponding serum DNA. Collectively these results confirm the fidelity of enrichment by Meth-DOP-PCR.

\section{Discussion}

We have described a simple procedure for the amplification of minute amount of DNA after sodium bisulfite treatment. This methodology, that we have named Meth-DOP-PCR, enables the accurate methylation analysis of DNA extracted from bodily fluids and is suited for the construction of

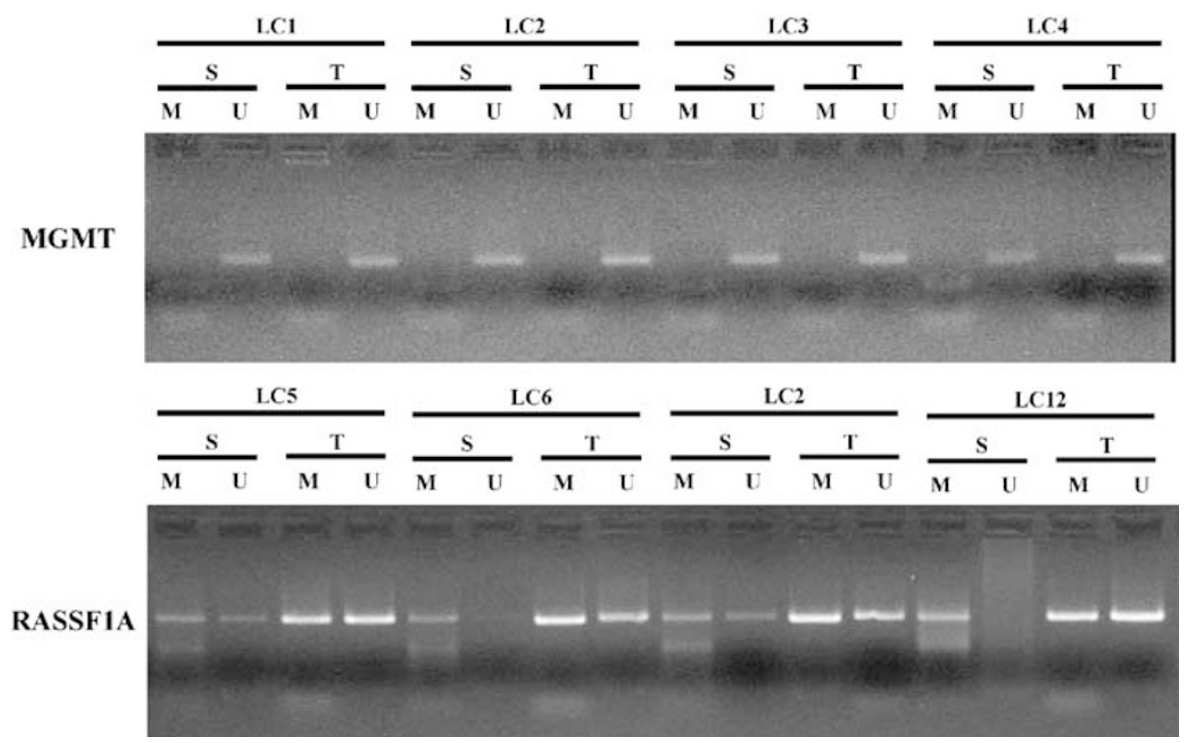

Figure 7 Analysis of the methylation status in paired tumor and serum DNA samples. Methylation analysis of the MGMT and RASSF1A gene promoters determined in paired samples of lung tumor (T) and circulating DNA (S) determined by Meth-DOP-PCR showing the concordance of the methylation status of these genes from the two DNA sources. 
methylation profiles from trace amount of DNA. In this respect we feel that Meth-DOP-PCR is a cost-effective methodology requiring common and inexpensive equipment and that can be utilized in conjunction with a variety of assays to assess the methylation status of multiple genes. In this initial description we have utilized Meth-DOP-PCR with the basic MSP procedure; however, this technique can be also coupled with MethyLight ${ }^{22}$ thus becoming a high-throughput procedure alternative to microarray-based technique ${ }^{23}$ for the large-scale methylation analysis in cancer cells.

In conclusion, we feel that Meth-DOP-PCR may be the basis for the development of novel assays for the early diagnosis of many types of cancer and for the patients follow-up.

\section{Acknowledgements}

We thank Dr Betta (Pathology Unit, ASO Alessandria) for providing the biological samples. This work was supported by grants from the Fondazione Italiana per la Lotta al Neuroblastoma, from the Associazione Italiana per la Ricerca sul Cancro (AIRC) and by the Ministero della Salute. I Gelvi is a fellow of the Fondazione Italiana per la Ricerca sul Cancro (FIRC), B Banelli is a Fellow of the Fondazione Italiana per la Lotta al Neuroblastoma.

\section{References}

1 Palmisano WA, Palmisano WA, Crume KP, et al. Predicting lung cancer by detecting aberrant promoter methylation in sputum. Cancer Res 2000;60:59545958.

2 Johnson PJ, Lo YMD. Plasma nucleic acids in the diagnosis and management of malignant disease. Clin Chem 2002;48:1186-1193.

3 Tsou JA, Hagen JA, Carpenter CL, et al. DNA methylation analysis: a powerful new tool for lung cancer diagnosis. Oncogene 2002;21:5450-5461.

4 Sozzi G, Conte D, Leon M, et al. Quantification of free circulating DNA as a diagnostic marker in lung Cancer. J Clin Oncol 2003;21:3902-3908.

5 Fujiwara K, Fujimoto N, Tabata M, et al. Identification of epigenetic aberrant promoter methylation in serum DNA is useful for early detection of lung cancer. Clin Cancer Res 2005;11:1219-1225.

6 Esteller M, Garcia-Foncillas J, Andion E, et al. Inactivation of the DNA-repair gene MGMT and the clinical response of gliomas to alkylating agents. New Engl J Med 2000;343:1350-1354.

7 Van Rijnsover M, Elsaleh H, Joseph D, et al. CpG Island methylator phenotype is an independent predictor of survival benefit from 5-fluorouracil in stage III colorectal cancer. Clin Cancer Res 2003;9:2898-2903.

8 Fiegl H, Millinger S, Mueller-Holzner E, et al. Circulating tumor-specific DNA: a marker for monitoring efficacy of adjuvant therapy in cancer patients. Cancer Res 2005;65:1141-1145.

9 Baylin SB, Herman JG. DNA hypermethylation in tumorigenesis. Trends Genet 2000;16:168-174.

10 Verma M, Srivatsava S. Epigenetics in cancer: implications for early detection and prevention. Lancet Oncol 2002;3:755-762.

11 Esteller M. Relevance of DNA methylation in the management of cancer. Lancet Oncol 2003;4:351-358.

12 Frommer M, McDonald LE, Millar DS, et al. A genomic sequencing protocol that yields a positive display of 5-methylcytosine residues in individual DNA strands. Proc Natl Acad Sci USA 1992;89:1827-1831.

13 Herman JG, Graff JR, Myohanen S, et al. Methylationspecific PCR: a novel PCR assay for methylation status of CpG islands. Proc Nat Acad Sci USA 1996;93: 9821-9826.

14 Esteller M, Sanchez-Cespedes M, Rosell R, et al. Detection of aberrant promoter hypermethylation of tumor suppressor genes in serum DNA from nonsmall cell lung cancer patients. Cancer Res 1999;59: 67-70.

15 Sadri R, Hornsby PJ. Rapid analysis of DNA methylation using new restriction enzyme sites created by bisulfite modification. Nucl Acid Res 1996;24: 5058-5059.

16 Telenius H, Carter NP, Bebb CE, et al. Degenerate oligonucleotide-primed PCR: general amplification of target DNA by a single degenerate primer. Genomics 1992;13:718-725.

17 Cheung VG, Nlson SF. Whole genome amplification using a degenerate oligonucleotide primer allows hundreds of genotypes to be performed on less than a nanogram of genomic DNA. Proc Natl Acad Sci USA 1996;93:14676-14679.

18 Grant SFA, Steinlicht S, Nentwich U, et al. SNP genotyping on a genome-wide amplified DOP-PCR template. Nucleic Acids Res 2002;30:e125.

19 Banelli B, Casciano I, Romani M. Methylation-independent silencing of the p73 gene in neuroblastoma. Oncogene 2000;19:4553-4556.

20 Di Vinci A, Perdelli L, Banelli B, et al. p16 ${ }^{\mathrm{INK} 4 \mathrm{a}}$ promoter methylation and altered protein expression in breast fibroadenoma and carcinoma. Int J Cancer 2005;114:414-421.

21 Banelli B, Gelvi I, Di Vinci A, et al. Distinct CpG methylation profiles characterize different clinical groups of neuroblastic tumors. Oncogene 2005;24: 5619-5628.

22 Eads CA, Danenberg KD, Kawakami K, et al. MethylLight: a high-throughput assay to measure DNA methylation. Nucleic Acids Res 2000;28:e32-e33.

23 Huang THM, Perry MR, Laux DE. Methylation profiling of $\mathrm{CpG}$ islands in human breast cancer cells. Human Mol Genet 1999;8:459-470. 